\title{
Innovative Initiatives in Control Education at Ryerson Polytechnic University: Fuzzy-Logic Control of the 3D-Helicopter Simulator
}

\section{Malgorzata S. Zywno}

Ryerson University

\section{Derek Pereira}

Ryerson University

Zywno, M. S., \& Pereira, D. (2000). Innovative initiatives in control education at Ryerson Polytechnic University: Fuzzy-logic control of the 3D-helicopter simulator. Proceedings of the 2000 American Control Conference. ACC (IEEE Cat. No.00CH36334), 6, 3991-3995.

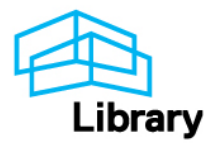


Proceedings of the American Control Conference

Chicago, llinois - June 2000

\title{
Innovative Initiatives in Control Education at Ryerson Polytechnic University - Fuzzy-Logic Control of the 3D-Helicopter Simulator
}

\author{
Malgorzata S. Zywno, M.Eng., Professor, Department of Electrical and Computer Engineering, Ryerson \\ Polytechnic University, Toronto, and Derek Pereira, B.Eng., GE Multilin, Markham, Canada \\ Email: gosha@ee.ryerson.ca, Derek.Pereira@indsys.ge.com
}

\begin{abstract}
This paper describes one of projects undertaken at Ryerson Polytechnic University to integrate emerging trends in control engineering into the undergraduate curriculum. An intelligent control scheme based on fuzzy-logic, and developed for an experimental setup, is discussed. The process is a highly coupled 8th order, multiple input - multiple output, 3 degrees-of-freedom simulator of a helicopter. Currently the setup is used to develop control strategies for undergraduate thesis students. Eventually the process will also be accessible to students remotely over the World Wide Web. The controller performance with the fuzzy-logic controller (FLC) is benchmarked against that of a conventional controller. The simulations show that the system performance under fuzzy-logic control does not deteriorate away from the equilibrium point and remains comparable with, or superior to, the performance under the linear control, over the whole range of operating conditions of this setup.
\end{abstract}

Key Words - Real-time Experiments, Simulation, Intelligent vs. Conventional Control, PID Controllers, Fuzzy-Logic.

\subsection{Introduction}

Modern control, which proves invaluable for providing solutions to well-structured aerospace problems, has not been as successful in process control applications. Industry continues to rely on $P I D$ (Proportional-Integral-Derivative) controllers [1]. Since PID controllers cannot always satisfy demands of complex modern plants, the search for new approaches has led to emergence of intelligent control, including fuzzy-logic. Over the past 20 years there have been many encouraging developments in intelligent control applications as an alternative to PID controllers, although control engineers are still generally reluctant to utilize them $[1,2]$.

With its wide range of applications in industry, process control remains an essential component of the Electrical and Computer Engineering program curriculum. Classical control with its emphasis on industrial process control, and particularly PID controllers, has traditionally been the first, and perhaps the only, course in control systems theory which all students in the program are required to take. The potential that intelligent control holds for process control is still rarely reflected in the undergraduate curriculum [3] and mostly remains in the domain of graduate studies [2]. Feasibility of undergraduate exposure to intelligent control concepts needs to be investigated.

\subsection{Control Systems Education at Ryerson}

The mandate of Ryerson Polytechnic University is to focus on the provision of a strong applied, and innovative, undergraduate education. The Department of Electrical and Computer Engineering offers one compulsory course in Classical Control, along with elective courses in Modern Control, Discrete Control, Robotics and System Models and Identification. Several projects to incorporate new trends in control engineering into teaching are under way.

The overall aim is to provide an educational experience involving theory and practical implementation in the undergraduate curriculum as well as in professional development [4]. In the classroom, practical aspects of control are emphasized. The visualization of theory and real-life examples are incorporated through the use of new media-enabled instruction, utilizing the Web, interactive simulations, Java applets and video [5]. Real-time experiments for the Control Lab [6], and realistic computer simulations are an integral part of instruction. 
One of the initiatives involves a real-time control setup (3D-Helicopter ${ }^{1}$ simulator shown in Figure 1) to develop challenging control strategies for students working on their undergraduate theses.

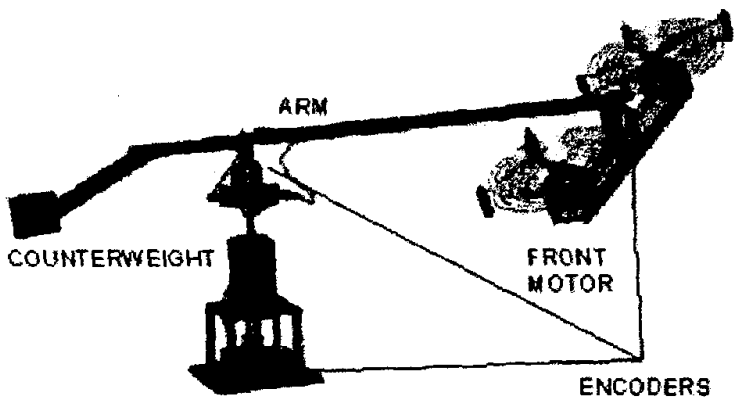

Figure 1: 3D Helicopter developed by Quanser Consulting, Inc.

Two projects have been completed so far. The first one, involving intelligent control using fuzzy-logic for the helicopter [7], is described at length in this paper. The second project developed a remote control scheme allowing accessing the helicopter over the World Wide Web through a Java interface. Remote access to the helicopter, normally available only to thesis students, will allow implementing linear control schemes developed in the Modern Control elective course as well.

\subsection{Project Background}

The objective of the project was to apply the fuzzy-logic control scheme to the helicopter Simulink simulation and to obtain the system performance equal to, or exceeding, the benchmarks established for a conventional control scheme. Since intelligent control is not part of the undergraduate curriculum, one of the challenges of the project was in the required independent study of literature, and in a quick ramping up of the practical experience in application of learned principles in computer simulations [7].

\subsection{Helicopter Setup}

The helicopter body is mounted at the end of an arm as shown in Figure 1 and is free to move about an elevation axis, a horizontal travel axis (a full $360^{\circ}$

\footnotetext{
${ }^{1}$ The 3D Helicopter setup was developed by Quanser Consulting, Inc. (http://www.quanser.com)
}

range of motion), as well as about a pitch axis. Angles of movement are measured via optical encoders. Two DC motors with propellers generate a force proportional to the voltage output of a controller that, if properly designed, will allow for a desired helicopter elevation and a desired travel rate or position to be achieved.

A data acquisition board (MultiQ) supplies a virtual link between WinCon ${ }^{2}$, a Windows' 95 -based real-time server on a $\mathrm{PC}$, and the helicopter hardware. WinCon is cross compatible with MATLAB, Simulink and Real-Time Workshop ${ }^{3}$. The helicopter is thus controlled in real-time by WinCon, any changes of the controller structure or its parameters are implemented by modifications in Simulink simulation diagrams and the $\mathrm{C}$-code is generated automatically. Such process is referred to as rapid control prototyping through hardware-in-the-loop simulation. It is a current and growing trend in control engineering.

\subsection{Quanser Controller}

The helicopter has three degrees of freedom: elevation, travel and pitch axes. Since travel is coupled with pitch, only two command signals are required. Travel/pitch and elevation commands are accessible with up-down and left-right signals from a joystick.

To apply the force in the travel direction, the helicopter body has to be pitched. A controller with the ability to limit the pitch angle is required, because steep pitching of the body may cause an altitude loss when the effective force required to keep the helicopter body elevated is diverted towards the travel and pitch angles. Thus three separate PID controllers (one for each of the motion axes) were initially implemented in the Quanser setup. Conventional PID tuning turned out to be inadequate for this complex system, resulting in a poor performance [8]. Most notably, changes in travel command visibly affected the elevation (coupling effect). Controller gains had to be therefore optimized, using a detailed linearized system model.

A complete derivation of the model is presented in [8]. The system dynamics are described by three highly nonlinear and coupled differential equations. The

\footnotetext{
${ }^{2}$ MultiQ and WinCon are registered trademarks of Quanser Consulting, Inc.

${ }^{3}$ MATLAB, Simulink and Real-Time Workshop are registered trademarks of MathWorks, Inc. (http://www.mathworks.com)
} 
system equations were linearized about an equilibrium point corresponding to a hover position, where the force generated by the motors and the body weight are balanced by a counterweight. The equations were then written in a standard state space form, with their coefficients evaluated using MAPLE [8]. The eight states were defined as elevation, pitch and travel angles, their derivatives, and elevation and travel integrals (for tracking of input commands). The controller outputs are voltages of front and back motors. State feedback gains are found using a Linear Quadratic Regulator (LQR) algorithm.

It is shown in [8] that the LQR controller can be implemented using the general PID structure and thus still effectively limit the pitch angle. The system response was much improved and the effect of the system coupling was minimized. However, an inherent problem still existed - since the design is optimized for the model linearized about the equilibrium point, the system tracking response deteriorates away from the equilibrium point.

Thus, the objective for the intelligent control scheme was to have a robust response both around, and away from, the equilibrium point. In order to evaluate the fuzzy-logic controller performance, benchmarking of the conventional control scheme developed by Quanser was first performed, by replacing the joystick with a pre-set computergenerated input, and the existing process-controller simulation model was tested for reliability.

\subsection{Furzy Logic Controller}

Fuzzy logic controller employs fuzzy sets, or membership functions, to represent its variables. The process of assigning membership functions to fuzzy variables is called fuzzification. For example, fuzzy sets of a triangular type were used for the elevation controller input variables (elevation error and speed) and the output variable (voltage applied to the motors). The controller was designed using Simulink and MATLAB Fuzzy Control Toobox. Figure 2 shows fuzzy sets for the elevation control.

The controller output is computed by using a fuzzy-logic rule base. The rule base is a linguistic set of conditional if...then statements, describing causal relationships between controller inputs and outputs. Table 1 shows the rule base for elevation control, consisting of 23 rules. The fuzzy rule base for travel control contains 298 rules. Its normalized surface is shown in Figure 3. A defuzzification process converts the fuzzy output variable to its crisp numerical value. The defuzzification method used in the project was a centroid method. In this approach, the crisp output value is calculated as a center of gravity of the area confined by the membership function curve.
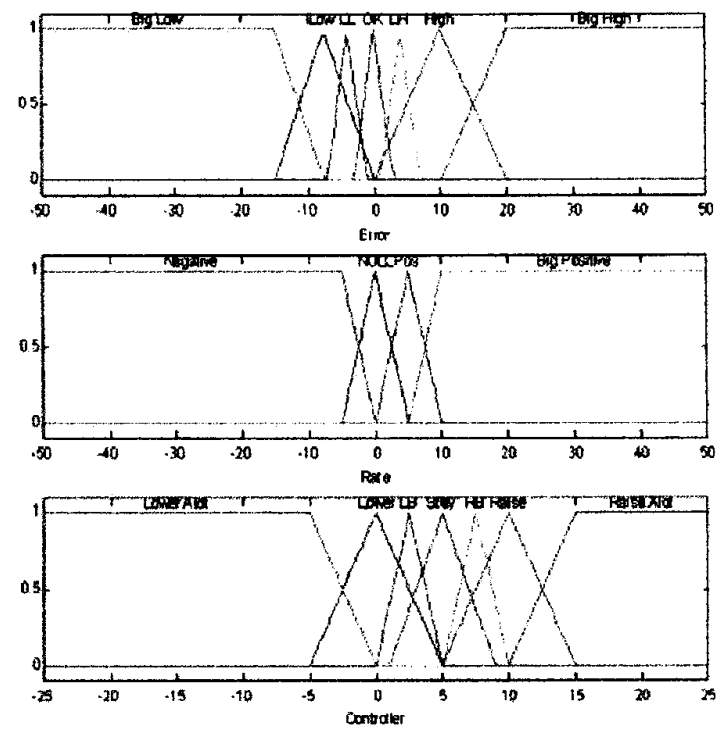

Figure 2: Fuzzy Sets Used for Elevation Control

Table 1: Rule Base for Elevation Control

$$
\begin{aligned}
& \text { 1. If (Error is Big.Low) and (Rate is Negative) then (Controller is Raise) (1) } \\
& \text { 2. If (Error is Big.Low) and (Rate is NULL) then (Controller is Raise.Alot) (1) } \\
& \text { 3. If (Error is Big.Low) and (Rate is Pos) then (Controller is Raise.Alot) (1) } \\
& \text { 4. If (Error is Big.Low) and (Rate is Big. Positive) then (Controllar is Stey) (1) } \\
& \text { 5. If (Error is Low) and (Rate is Negative) then (Controller is Reise) (1) } \\
& \text { 6. If (Error is Low) and (Rate is NULL) then (Controller is Raise) (1) } \\
& \text { 7. If (Error is Low) and (Rate is Pos) then (Controller is Raise) (1) } \\
& \text { 6. If (Error is Low) and (Rate is Big.Positive) then (Controller is Lower) (1) } \\
& \text { 9. If (Error is OK) and (Rate is Negative) then (Controller is Raise) (1) } \\
& \text { 10. If (Error is OK) and (Rate is NULL) then (Controller is Stay) (1) } \\
& \text { 11. If (Error is OK) and (Rate is Pos) then (Controller is Lower) (1) } \\
& \text { 12. If (Error is OK) and (Rate is Big.Positive) then (Controller is Lower) (1) } \\
& \text { 13. If (Error is High) and (Rate is Negative) then (Controller is LB) (1) } \\
& \text { 14. If (Error is High) and (Rate is NULL) then (Controller is Lower Alot) (1) } \\
& \text { 15. If (Error is High) and (Rate is Pos) then (Controlier is Stay) (1) } \\
& \text { 16. If (Error is Big. High) and (Rate is Negative) then (Controller is Stay) (1) } \\
& \text { 17. If (Error is Big.High) and (Rete is NULL) then (Controller is Lower.Alot) (1) } \\
& \text { 18. If (Error is Big.High) and (Rate is Pos) then (Controller is Raise) (1) } \\
& \text { 19. If (Error is LH) and (Rate is Negative) then (Controller is Stay) (1) } \\
& \text { 20. If (Error is LH) and (Rate is NULL) then (Controller is Lower.Alot) (1) } \\
& \text { 21. If (Error is LL) and (Rate is Pos) then (Controller is Stey) (1) } \\
& \text { 22. If (Error is LL) and (Rate is NULL) then (Controller is R日) (1) } \\
& \text { 23. If (Error is LL) and (Rate is Negative) then (Controller is Raise.Alot) (1) }
\end{aligned}
$$

\subsection{Simulations and Results}

Figure 4 shows the complete controller structure for the helicopter simulation. For reliability, the real 
system response was measured and compared with the LQR model-controller simulations, which proved to be very accurate, as shown in Figure 5.

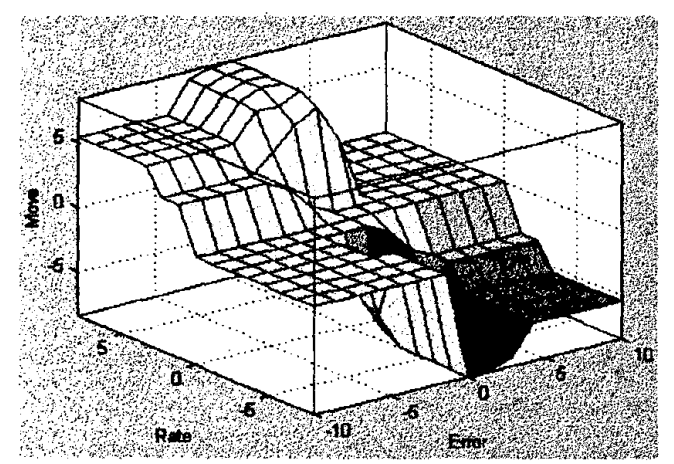

Figure 3: Fuzzy Rule Surface for Travel Control

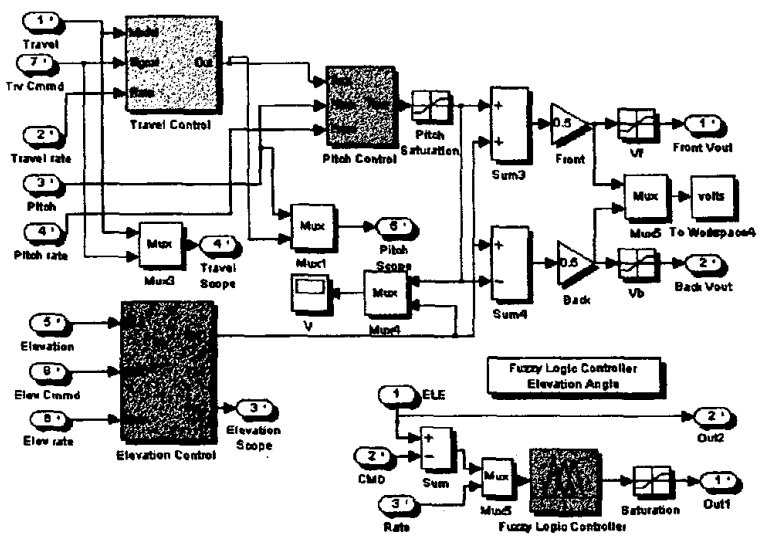

Figure 4: Fuzzy Logic Control of 3D-Helicopter
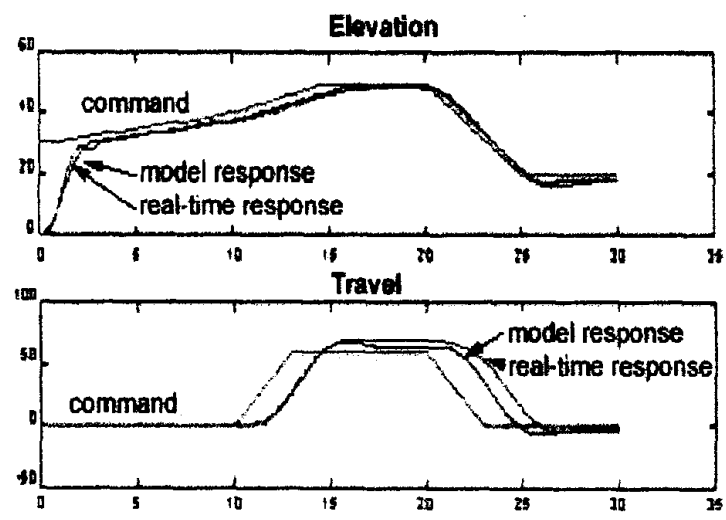

Figure 5: Model - Simulations vs. Real Response

These simulations were then used as a benchmark for the fuzzy-logic controller. To evaluate the FLC performance, its response to preset elevation and travel commands was simulated, and compared with the conventional (LQR) controller response simulations. Tables 2 and 3 show this comparison with respect to the following specifications: percent overshoot, rise time, and steady state error.

Table 2: FLC vs. LQR Performance - Elevation

\begin{tabular}{|l|c|c|c|c|}
\cline { 2 - 5 } \multicolumn{1}{c|}{} & \multicolumn{1}{c|}{ SLOPE } & \multicolumn{1}{c|}{ UP } & \multicolumn{1}{c|}{ SLOPE } & \multicolumn{1}{c|}{ DOWN } \\
\cline { 2 - 5 } \multicolumn{1}{c|}{} & LQR & FLC & LQR & FLC \\
\hline PO & $0.63 \%$ & $0.37 \%$ & $17.5 \%$ & $8.0 \%$ \\
\hline Trise & $2.14 \mathrm{~s}$ & $1.37 \mathrm{~s}$ & $1.56 \mathrm{~s}$ & $1.12 \mathrm{~s}$ \\
\hline E $_{s s}$ & $0.05^{\circ}$ & $0.05^{\circ}$ & $0.5^{\circ}$ & $0.1^{\circ}$ \\
\hline
\end{tabular}

Table 3: FLC vs. LQR Performance - Travel

\begin{tabular}{|l|c|c|c|c|}
\cline { 2 - 5 } \multicolumn{1}{c|}{} & \multicolumn{1}{c|}{ SLOPE } & \multicolumn{1}{c|}{ DOWN } & SLOPE & \multicolumn{1}{c|}{ UP } \\
\cline { 2 - 5 } \multicolumn{1}{c|}{} & LQR & FLC & LQR & FLC \\
\hline PO & $6.0 \%$ & $1.8 \%$ & $1.4 \%$ & $2.2 \%$ \\
\hline T $_{\text {rise }}$ & $1.21 \mathrm{~s}$ & $0.6 \mathrm{~s}$ & $1.61 \mathrm{~s}$ & $1.0 \mathrm{~s}$ \\
\hline E $_{\text {ss }}$ & $1.1^{\circ}$ & $0.15^{\circ}$ & $0.2^{\circ}$ & $0.05^{\circ}$ \\
\hline
\end{tabular}

The fuzzy-logic controller consistently outperforms the conventional controller as shown in Tables 2 and 3 and in Figures 6 and 7.

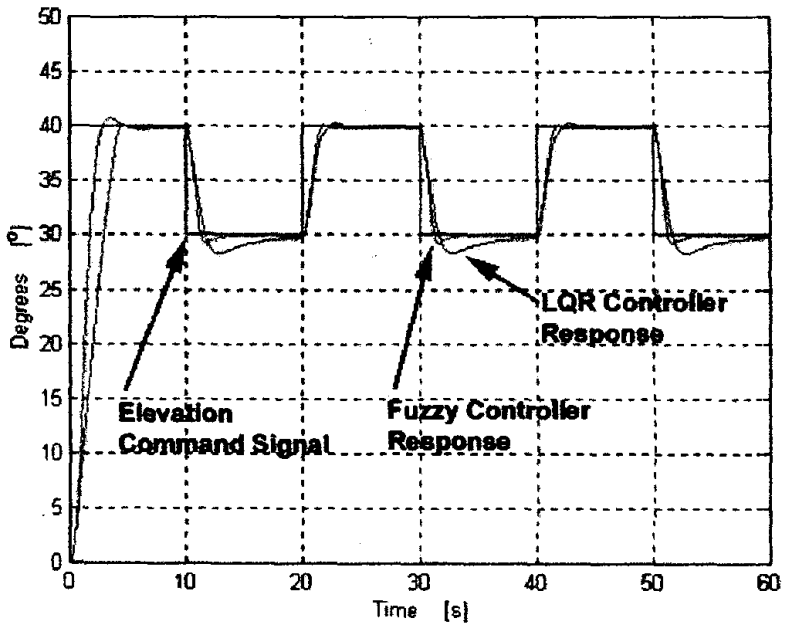

Figure 6: FLC vs. LQR Response - Elevation 
Moreover, as seen in Figure 7 , the FLC performance remains robust, i.e. relatively unchanged both close to, as well as away from, the equilibrium point.

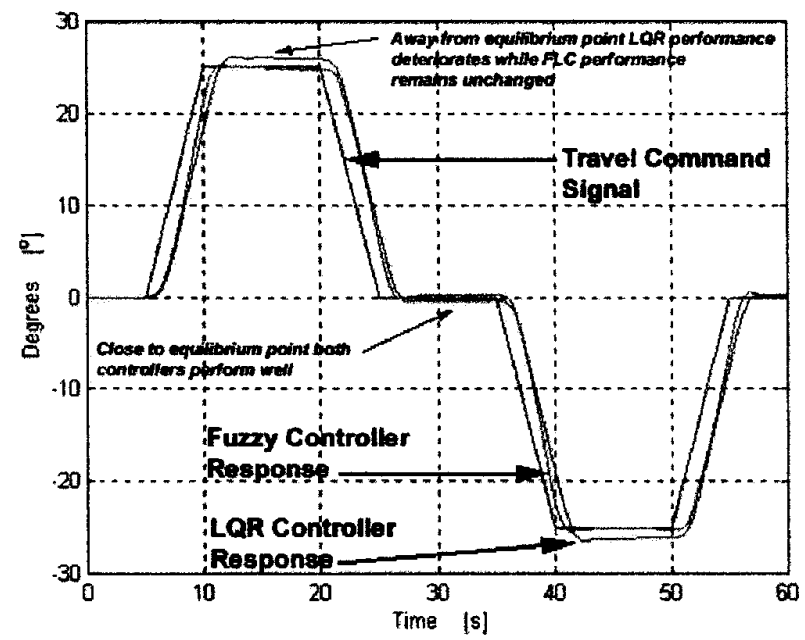

Figure 7: FLC vs. LQR Response - Travel

\subsection{Recommendations}

While the elevation controller required only 23 rules, when both elevation and travel controllers were running at the same time, 321 rules had to be processed for every position element, resulting in excessive simulation times! Therefore, the existing rule base has to be optimized before a real-time implementation of this fuzzy-logic controller becomes viable. The rule base optimization and the real-time implementation will be the subject of a new project.

Another important issue is a degree of confidence in the system model when relying entirely on simulation results. Developing a robust model to represent system dynamics over a wide range of operating conditions is a challenging problem in itself. Artificial neural networks (ANN) may provide an alternative to a tedious conventional modeling. Such model could then be used to reliably test different control strategies.

\subsection{Conclusions}

The designed fuzzy logic controller not only meets the objective of controlling the helicopter to achieve stable tracking of various inputs but is superior in performance when contrasted with the conventional linear controller simulations. A relatively short time frame in which this project was accomplished indicates that inclusion of some aspects of intelligent control into Ryerson undergraduate curriculum is quite realistic.

\subsection{Acknowledgements}

The authors would like to express their gratitude to Dr. Jacob Apkarian of Quanser Consulting, Inc., for his generous support of this project as well as of other control systems initiatives undertaken in the Department of Electrical and Computer Engineering at Ryerson.

\subsection{Bibliography}

1. King, R.E., Computational Intelligence in Control Engineering, Marcel Dekker, Inc., 1999.

2. Yurkovich, S. and Passino, K.M., A Laboratory Course on Fuzzy Control, IEEE Trans. Edu., Vol. 42, No.1, February 1999, pp. 15-21.

3. Saneifard, S., Prasad, N.R. , Smolleck, H.A. and Wakileh, J.J., Fuzzy-Logic Based Speed Control of a Shunt DC Motor, IEEE Trans. Edu., Vol. 41, No.2, May 1998, pp. 159-164.

4. Zywno, M.S. and Kennedy, D.C., An Initiative at Ryerson Polytechnic University in Meeting the Demands of Industry - Development of a Multimedia Enhanced Short Course in Practical PID Control for Engineers and Technologists, Workshop at the 1998 ASEE/IEEE Frontiers in Education Conference, Tempe, Arizona.

5. Zywno, M.S., Brimley, W. and White, W.E., Effective Integration of Multimedia Courseware as an Enhancement of Learning Experiencs in Engineering Education, Proc. of 2nd UICEE Annual Conference on Engineering Education, Auckland, New Zealand, 1999, pp. 37-41.

6. Zywno, M.S. and Kennedy, D.C., A Practical Servomotor Project: Combining the Web with Simulation Tools to Solidify Concepts in Undergraduate Control Education, Proc. of the American Control Conference (ACC), Philadelphia, USA, 1998, Vol. 2, pp. 1309-1313.

7. Pereira, D., Fuzzy Logic Controller for $3 D$ Helicopter Model, B.Eng. Thesis, Ryerson Polytechnic University, May 1999.

8. Apkarian, J., 3D Helicopter Experiment Manual, Quanser Consulting, Inc., 1998. 